\title{
Transjugular Intrahepatic Portosystemic Shunt (TIPS) versus Balloon-occluded Retrograde Transvenous Obliteration (BRTO) for the Management of Gastric Varices
}

\author{
Wael E. A. Saad, M.D., F.S.I.R., ${ }^{1}$ and Michael D. Darcy, M.D., F.S.I.R. ${ }^{2}$
}

Variceal bleeding is one of the major complications of portal hypertension. Gastric variceal bleeding is less common than esophageal variceal bleeding; however, it is associated with a high morbidity and mortality rate and its management is largely uncharted due to a relatively less-established literature. In the West (United States and Europe), the primary school of management is to decompress the portal circulation utilizing the transjugular intrahepatic portosystemic shunt (TIPS). In the East (Japan and South Korea), the primary school of management is to address the gastric varices $(\mathrm{GVs})$ specifically by sclerosing them utilizing the balloon-occluded retrograde transvenous obliteration (BRTO) procedure. The concept (1970s), evolution, and development (1980s-1990s) of both procedures run parallel to one another; neither is newer than the other is. The difference is that one was adopted mostly by the East (BRTO), while the other has been adopted mostly by the West (TIPS). TIPS is effective in emergently controlling bleeding for GVs even though the commonly referenced studies about managing GVs with TIPS are studies with TIPS created by bare stents. However, the results have improved with the use of stent grafts for creating TIPS. Nevertheless, TIPS cannot be tolerated by patients with poor hepatic reserve. BRTO is equally effective in controlling bleeding GVs as well as significantly reducing the GV rebleed rate. But the resultant diversion of blood flow into the portal circulation, and in turn the liver, increases the risk of developing esophageal varices and ectopic varices with their potential to bleed. Unlike TIPS, the blood diversion that occurs after BRTO improves, if not preserves, hepatic function for 6-9 months post-BRTO. The authors discuss the detailed results and critique the literature, which has evaluated and remarked on both procedures. Future research prospects and speculation as to the ideal patients for each procedure are discussed.

KEYWORDS: History, BRTO, transvenous obliteration, varices, TIPS, rebleeding

${ }^{1}$ Division of Vascular Interventional Radiology, Department of Radiology, University of Virginia Health System, Charlottesville, Virginia; ${ }^{2}$ Division of Interventional Radiology, Mallinckrodt Institute of Radiology, Washington University, St. Louis, Missouri.

Address for correspondence and reprint requests: Wael E. A. Saad, M.D., F.S.I.R., Department of Radiology and Medical Imaging, University of Virginia Health System, Box 800170, 1215 Lee Street, Charlottesville, VA 22908 (e-mail: wspikes@yahoo.com).
Management of Gastric Varices: Endoscopic, BRTO, and TIPS; Guest Editors, Wael E. A. Saad, M.D., F.S.I.R., and Saher S. Sabri, M.D.

Semin Intervent Radiol 2011;28:339-349. Copyright (C) 2011 by Thieme Medical Publishers, Inc., 333 Seventh Avenue, New York, NY 10001, USA. Tel: +1(212) 584-4662.

DOI: http://dx.doi.org/10.1055/s-0031-1284461.

ISSN 0739-9529. 
Objectives: Upon completion of this article, the reader should be able to identify the outcomes and limitations of both the transjugular intrahepatic portosystemic shunt (TIPS) and balloon-occluded retrograde transvenous obliteration (BRTO) procedures in managing gastric varices; state the advantages and disadvantages of both procedures and the significant limitation of the literature regarding the subject of minimal invasive management of gastric varices especially when utilizing stent grafts for TIPS in patients with gastric variceal bleeding.

Accreditation: Tufts University School of Medicine is accredited by the Accreditation Council for Continuing Medical Education to provide continuing medical education for physicians.

Credit: Tufts University School of Medicine designates this journal-based CME activity for a maximum of 1 AMA PRA Category 1 Credit $^{\mathrm{TM}}$. Physicians should claim only the credit commensurate with the extent of their participation in the activity.

Minimally invasive procedural management of portal hypertension, whether endoscopic or imageguided, is at the forefront in the management of portal hypertension complications. Variceal bleeding is one of the major complications of portal hypertension. Esophageal variceal bleeding is more common and the role of minimally invasive procedures is well established and charted. ${ }^{1}$ Gastric variceal bleeding is less common, however, and is associated with high morbidity and mortality rates ( $45-55 \%$ mortality); its management is largely uncharted due to a relatively less mature/lessestablished literature. ${ }^{1-4}$ Furthermore, endoscopic management of gastric varices is usually less effective when compared with its role in the management of esophageal varices. ${ }^{4-8}$

From an interventional radiology (minimally invasive image-guided procedures) standpoint there is a controversy at the international level as to the ideal management of gastric varices. ${ }^{3,9-12}$ In the West (United States and Europe), the primary school of management is to decompress the portal circulation utilizing the transjugular intrahepatic portosystemic shunt (TIPS). This is in line with the long history of decompressive surgeries (surgical portosystemic shunts) that were more popular prior to the advent of the TIPS procedure. In the East (Japan and South Korea), the primary school of management is to address the gastric varices specifically by sclerosing them utilizing the balloon-occluded retrograde transvenous obliteration (BRTO) procedure. Unfortunately, the spontaneous gastrorenal/gastrosplenorenal shunt (a natural portosystemic decompressive shunt) is occluded during the BRTO procedure; ${ }^{9,10,13}$ thus there is aggravation of the portal hypertension, ${ }^{14-17}$ which is contrary to the Western decompressive ideology/school of portal hypertension management. Herein lies the center of the controversy: to decompress or not to decompress; to manage portal hypertension globally, or to specifically address the complication at hand.

Here we discuss the controversy between TIPS and BRTO and display and critique the available outcome data of both procedures. Out of the limited data available, speculation of future research and clinical trends are also made.

\section{THE CONCEPT AND HISTORY OF THE TIPS AND BRTO PROCEDURES}

One cannot discuss these two procedures (BRTO and TIPS) without addressing the clear East versus West geographic divide between them. To understand this dichotomy to impartially critique these procedures, one must understand the history and evolution of these two, very involved, but different procedures.

\section{The Concept and History of the BRTO Procedure}

Transvenous obliteration is actually an older idea and was practiced in the mid-1970s in the pretransjugular intrahepatic portosystemic shunt (TIPS) era as an interventional radiology procedure for the management of bleeding esophageal and gastroesophageal varices from a percutaneous transhepatic approach. ${ }^{18-22}$ These percutaneous transhepatic obliterations were mostly performed utilizing coils, Gelfoam, and/or sclerosants (such as absolute alcohol and 30-50\% glucose solution). ${ }^{18-22}$ These transhepatic procedures were usually performed without utilizing occlusive balloons that intend to modulate blood flow. ${ }^{18-22}$ The initial transhepatic obliterative experience in the 1970s was short lived due to relatively poor clinical success ${ }^{18-22}$ and ultimately the advent of the TIPS procedure in the early 1990s (see below).

The concept of balloon-occluded retrograde transvenous obliteration (BRTO) as we know it today is accessing the portosystemic gastrorenal shunt via the left renal vein from a transjugular or transfemoral approach. ${ }^{9,10,13,23}$ Most authors refer to Kanagawa et al (1991-1993) as the inventor of BRTO. ${ }^{13}$ However, the first published document of an attempt at balloonoccluded sclerotherapy of the gastrorenal shunt for the management of gastric varices was authored by Olson et al in 1984 out of Indiana University. ${ }^{23}$ This is clearly 5 and 7 years prior to the commencement of the BRTO clinical practice (1991) and publication (1993), respectively, of Kanagawa et al. ${ }^{13}$ Olson et al utilized a transfemoral balloon occlusion catheter and absolute alcohol for the successful sclerosis attempt. ${ }^{23}$ Embolic coils were also placed in the outflow gastrorenal shunt. ${ }^{23}$ The term used for the procedure was "transrenal-vein reflux ethanol sclerosis" and not BRTO. ${ }^{23}$ 
Subsequently, Kanagawa et al revived the BRTOconcept and developed the BRTO-procedure in the early 1990s, coining the term, balloon-occluded retrograde transvenous obliteration (B-RTO). ${ }^{13}$ They utilized ethanolamine oleate as an endovascular sclerosant which Western (European and American) interventional radiologists were not familiar with. Ethanolamine oleate is an established upper endoscopy variceal sclerosant. However, when used from an endovascular standpoint, there is a greater risk for hemolysis, hemoglobinuria, and potentially hemoglobin-induced renal tubular dysfunction. ${ }^{9,10}$ The antidote of free hemoglobin is haptoglobin, which conjugates with it. Haptoglobin is available in Japan and is not available for human and commercial use in the United States. In fact, haptoglobin is routinely given, 2000 to (more commonly given) 4000 units, as an intravenous infusion with all ethanolamine oleate BRTO procedures in Japan., ${ }^{3,9-13,24-42}$ Consequently, the Japanese developed, evolved, and clinically applied this procedure. ${ }^{3,9-13,24-42}$ They took it to the clinical level and established it as a viable and successful procedure that it is today. ${ }^{3,9-13,24-42}$

There are two main hypotheses why BRTO has not been clinically practiced (until recently) in the United States. The first is the unfamiliarity of interventional radiologists in the United States with the BRTOprocedure as well as the well-described (in Japan and Korea) sclerosant: ethanolamine oleate. In addition, the lack of the antidote, haptoglobin (see above), potentially concerned many radiology interventionalists in using ethanolamine oleate. The second cause of the delayed utilization of BRTO in the United States is that the ideology or thought process of managing portal hypertension complications in the United States is decompression and reducing the portal pressure. It is not, as is the case with the BRTO procedure, mere management of a particular complication (in this case, potentially bleeding gastric varices) with the added risk of aggravating the portal hypertension and potentially having patients subsequently develop other complications of portal hypertension such as potentially bleeding esophageal varices and/or ascites. BRTO is known to cause aggravation of esophageal varices and may increase the risk of developing ascites (please see "Balloon-occluded Retrograde Transvenous Obliteration (BRTO): Technical Results and Outcomes" in this issue). ${ }^{3,9-13,24-42}$ In addition, reinforcing the theory of decompression being favored over sclerosis, is the initial experience of transhepatic obliteration in Europe and the United States did not have a satisfactory technical, hemodynamic or clinical success. ${ }^{18-22}$ The technical success of these early transhepatic procedures varied from $54 \%$ to $88 \%$ with a rebleed rate of successful cases of $29-86 \%$ and an overall intent-to-treat clinical success rate of $25-31 \%$ and an image based variceal recannulation of over $80 \%{ }^{18-20}$ (please compare with theBRTO results in
"Balloon-occluded Retrograde Transvenous Obliteration (BRTO): Technical Results and Outcomes" in this issue).

\section{The Concept and History of the TIPS Procedure}

The concept of the transjugular intrahepatic shunt (TIPS) is, as the name implies, creating an intrahepatic connection between the portal vein and the hepatic outflow vein. ${ }^{4-50}$ The first published experimental study in animals (canines) was by Burgener et al in $1979 .{ }^{43}$ They performed the connection with balloon angioplasty of the tract without stent placement. ${ }^{43}$ Dr. Burgener was a Swiss radiologist who practiced at the University of Rochester (Rochester, NY) and published more of these experimental animal studies in the 1980s. ${ }^{43-46}$ The first published experimental TIPS animal study utilizing stents (Gianturco stents) was by Rosch et al from the Dotter Institute (Portland, OR) in $1987 .{ }^{47}$ The actual practice of creating a TIPS in humans started in Europe and the United States ca. 1989-1990, ${ }^{48-51}$ with early reports of TIPS in humans published from 1991 to $1992 .^{48-50}$ The first sizeable reports of TIPS (number of patients $>40$ ) in humans with commercially available stents (Wallstents and Strecker stents) was in 1993$1994 .{ }^{52,53}$

The transjugular intrahepatic portosystemic shunt (TIPS) was hindered by three main problems: patency, hepatic encephalopathy, and the toll it has on hepatic function. Longevity (patency) of the TIPS was a primary problem and disease recurrence was strongly related to lack of TIPS patency (TIPS dysfunction: stenosis or occlusion).${ }^{54-57}$ Experimentation on animal models in the 1990s demonstrated that most TIPS stenoses and possibly occlusions were due to biliary-TIPS fistulas. ${ }^{58,59}$ The biliary leaks induced pseudointimal hyperplasia and the bile was also thrombogenic. ${ }^{58,59}$ To counter this, the solution was creation of the shunt utilizing covered stents. ${ }^{58,59}$ Experimentation with silicone-covered and expanded polytetrethylene- (e-PTFE-) covered stents showed superiority of the e-PTFE covered TIPSstents. ${ }^{58-60}$ From 2000 to 2003, early human reports on e-PTFE covered stent placements were published. Most reports were an amalgamation of de novo TIPS creation and TIPS revisions utilizing e-PTFE stentgrafts. ${ }^{60-63}$ As of 2003, reports of de novo TIPS creation with numbers of human patients started to be reported from Europe. ${ }^{64-73}$ These reports confirmed the clear superiority of e-PTFE stent grafts compared with bare stents ${ }^{64-73}$ to the extent that the history of TIPS would be divided into the pre- and poststent-graft era. ${ }^{74}$ However, there remains the two other problems with TIPS: the increased incidence of encephalopathy, and thetoll that TIPS has on liver function. ${ }^{75-78}$ Furthermore, there is relatively new data accumulating from the United States and Europe regarding candidacy of 
patients for the TIPS procedure; where patients with poor hepatic reserve (MELD >17-19) have been shown to do poorly with the TIPS procedure. ${ }^{75-78}$

When discussing with Japanese interventional radiologists why the TIPS procedure is not commonly performed in Japan, they refer to the increased incidence of encephalopathy and post-TIPS hepatic dysfunction. In addition, they anecdotally refer to the fact that liver cirrhosis in Japanese patients presents with shrunken and hard livers which makes TIPS technically challenging (personal communication with Takashi Kitanosono, Showa University, Japan and Kenji Takizawa, St. Marianna University, Kawazaki, Japan, March 9, 2009).

\section{Summary of the History and Evolution of TIPS and BRTO}

The concept, evolution, and development of both procedures run parallel to one another; neither is more novel than the other is. The concept of both procedures dates back to the 1970s with early experimentation in the 1980s. They both began to be performed on humans in the 1990s and matured to be effective and established procedures in the last decade. The difference is that one has been adopted mostly by the East (BRTO) and the other has been adopted mostly by the West (TIPS). They both were conceived from a certain managerial ideology and through their clinical success, they further engraved that ideology.

\section{OUTCOMES OF THE TIPS AND BRTO PROCEDURES FOCUSED ON THE MANAGEMENT OF GASTRIC VARICES}

There is actually limited data in the literature that is specific to TIPS for gastric varices (six studies). ${ }^{26,79-83}$ Most, even more recent studies, amalgamate all varices (vast majority esophageal, gastric, or gastroesophageal) and one cannot glean the outcomes specific to patients with gastric varices in these amalgamated studies. On the other hand, BRTO is a procedure that is specific to gastric variceal management and there is more data showing efficacy of the procedure (over 40 studies). ${ }^{3,9-13,24-42}$ From this standpoint alone, the TIPS procedure stands at a disadvantage.

\section{Outcome of TIPS for Patients with Gastric Varices}

There are six TIPS studies that address patients with portal hypertension complicated by gastric varices. ${ }^{26,79-83}$ These six studies evaluate 147 patients (range for individual studies: $12-35$ patients). ${ }^{26,79-83}$ Two of these studies have intrainstitutional comparisons with BRTO outcomes. ${ }^{26,79}$
Two studies were published before the year 2000 and had 60 patients with actively bleeding gastric varices who had undergone TIPS created by bare stents. ${ }^{80,81}$ The success of TIPS in controlling the active variceal bleeding was $94 \%(90-96 \%){ }^{80,81}$ However, the 6-7 month and 12 month rebleeding rate was $26-29 \%$ and $31 \%$, respectively. ${ }^{80,81}$ The post-TIPS hepatic encephalopathy rate was $16 \%{ }^{81}$

The four studies published in the last decade evaluated 87 patients with gastric varices who underwent a TIPS procedure. ${ }^{26,79,82,83}$ However, all the TIPS were still created with bare stents. ${ }^{26,79,82,83}$ The 12 - to 24- month post-TIPS rebleed rate was $11-20 \% .^{26,79,82,83}$ The improved rebleeding rate when comparing the preand post-year 2000 studies may be due to better techniques and improved clinical and imaging (Doppler ultrasound) surveillance and follow-up. The main problem with all six of these studies is that the transjugular intrahepatic portosystemic shunts were created with bare stents and not stent grafts. ${ }^{26,79-83}$ The use of stent grafts would probably make a significant difference considering that TIPS patency has improved from 30-69\% (bare stents) to 76-92\% (stent grafts) with the advent of the commercially available Viatorr stent graft (Gore \& Assoc., Flagstaff, AZ). ${ }^{64-74,84}$ This is particularly true when considering that over $70 \%$ of gastric variceal rebleeding after TIPS have been associated with TIPS dysfunction (TIPS stenosis or thrombosis). ${ }^{82}$ Furthermore, a common quote in BRTO studies when referring to the TIPS literature, is that the gastric variceal rebleed rate after TIPS is $50 \%$, referencing Sanyal et al. ${ }^{85}$ This is not a true statement. This is the gastric variceal resolution rate and not the rebleeding rate $(N=6 / 12) .{ }^{85}$ Moreover, four of the six unresolved gastric varices $(75 \%)$ had a preTIPS portosystemic gradient of $<12 \mathrm{~mm}$ mercury ${ }^{85}$ (see discussion below). Not surprising. Chao et al showed that gastric varices bleed at a lower hepatic-portal venous gradient (mean gradient 11.2 for gastric, 15.5 for esophageal). ${ }^{86}$

The two studies that had intrainstitutional comparison between BRTO and TIPS had 85 BRTO cases and 40 TIPS cases. $^{26,79}$ Unfortunately, the study by Choi et al had a small sample (BRTO: 8, TIPS: 13) that was too small for a statistical comparison. ${ }^{79}$ The rebleeding and encephalopathy rate was $15 \%$ versus $0 \%$ and $31 \%$ versus $0 \%$ for TIPS versus BRTO, respectively. ${ }^{79}$ The more significant study was by Ninoi et al and had a larger sample (BRTO: 77, TIPS: 27). ${ }^{26}$ The one year rebleeding rate was $20 \%$ versus $2 \%$ for TIPS versus BRTO, respectively $(p<.01) .{ }^{26}$ Furthermore, the 1,3 , and 5 year survival after BRTO was better than after TIPS ( $p=.01$ ): $96,83,76 \%$ versus $81,64,40 \%$, respectively. ${ }^{26}$ However, the improved survival for BRTO compared with TIPS was limited to patients who were classified preprocedurally as Child-Pugh A. There was no difference in survival for patients who were classified 
as Child-Pugh B or $\mathrm{C} .{ }^{26}$ Overall, the percentage of patients in both studies that experienced hepatic encephalopathy after TIPS was $19-31 \% .{ }^{26,79}$ The noncomparative study by Barange et al also showed a post-TIPS (all TIPS created for gastric varices) hepatic encephalopathy rate of $16 \% .^{81}$

\section{Outcome of BRTO for Patients with Gastric Varices}

Details of the technical results and outcomes are discussed in this issue in "Balloon-occluded Retrograde Transvenous Obliteration (BRTO): Technical Results and Outcomes." Overall the technical success of patients with gastrorenal shunts (noncandidates not included) for BRTO only ranges from $79-100 \%{ }^{3,11,12,25-32,35,39-41,87-90}$ Two studies clearly identified primary treatment of gastric varices with BRTO, reserving Balloon-occluded antegrade transvenous obliteration (BATO) via a percutaneous transhepatic route as a rescue., ${ }^{3,26}$ These two studies had a BRTO technical success without and with BATO rescue of $84-98 \%$ and $100 \%$, respectively. ${ }^{3,26}$ The effectiveness of BRTO in controlling bleeding gastric varices is $91-100 \%$ in two studies evaluating 20 patients $(N=19 / 20$, controlled: $95 \%))^{3,25}$ Procedural and long-term complications, when mentioned, are displayed in Table 1. 3 ,11,12,24,25,27-29,31,32,35,36,39,87-92

The aggravation of nongastric (esophageal or duodenal) appears to be a major problem in the longrun and is reflective of increasing portal hypertension following BRTO.,11,12,24,25,27,31,36,87,89-92 It varies widely probably depending on the degree of vigilance, documentation and thoroughness of follow-up endoscopy. However, in four main studies evaluating 160 patients who had undergone BRTO who had continuous endoscopic follow-up post-BRTO, the esophageal variceal aggravation rate (expressed as a Kaplan-Meier analysis) at 1, 2, and 3 years was $27-35 \%, 45-66 \%$, and 45-91\%, respectively. ${ }^{11,31,35,42}$ In another two studies evaluating 117 patients with BRTO, the percentage of patients with aggravated esophageal varices was $30-68 \%$ and the patients that had bleeding esophageal varices was $17-24 \%$ of patients (36-57\% of patients with aggravated esophageal varices went onto to bleeding). ${ }^{27,90}$ Again, one can argue that the percentage of esophageal variceal bleeding may be significantly reduced by a higher vigilance of endoscopic follow-up and more aggressive endoscopic therapy (esophageal banding and/or sclerotherapy). Other complications reflective of increased portal hypertension following BRTO are the development of portal hypertensive gastropathy (occurs in $5-13 \%$ ) and possibly ascites (occurs in 0-44\%) and hydrothorax/pleural effusion (occurs in $0-8 \%)^{28,31,32,35,39,88}$

The rebleeding rate following BRTO depends on how it is presented. We believe that there is a pressing need to standardize reporting of BRTO
Table 1 Procedural Complications of Balloon-occluded Transvenous Obliteration (BRTO) Utilizing Ethanolamine Oleate

Complication Type Incidence (\%)

Complication Type

$15-100 *$

All pulmonary embolism

$1.5-4.1$

Symptomatic pulmonary embolism

$1.4-2.5$

Cardiac arrhythmia

1.5

Anaphylaxis

$2.2-5.0$

Rapid / fulminant hepatic failure

$4.8-7.0$

Death within 30 days from fulminant

$0.0-4.1$

hepatic failure

Renal failure

4.8

Long-term Complications

Encephalopathy

$17.6^{\dagger}$

Portal hypertensive gastropathy

$5.3-13.2$

Post-BRTO gastropathy (not to extent

56.5

of portal hypertensive gastropathy)

Aggravation of esophageal varices

$14-68^{\ddagger}$

Bleeding from esophageal varices

$17-24^{\ddagger}$

Duodenal varices

Up to 3.2

Bleeding duodenal varices

Up to 2.3

Ascites

0-43.5

Spontaneous bacterial peritonitis

Up to 8.2

Pleural effusion (hydrothorax)

5.3-7.9

Portal vein thrombosis

Renal vein thrombosis (no clinical

Up to 4.7

Up to 5.0

\section{consequences)}

Based on data from references $3,11,12,24,25,27-29,31,32,35,36,39,87-92$

*This is a common complication and is usually without clinical consequences. The wide range is probably due to how well it is documented for the retrospective audit which most of studies are categorized as.

The rate of encephalopathy is subject to definition and how closely there was clinical follow-up and may vary widely. This is a feature of the entire portal hypertension interventional radiology literature and is not specific to the BRTO literature.

¥The rate of variceal aggravation is subject to definition and how closely there was endoscopic follow-up and may vary widely. In addition (anecdotally), aggressive pre-BRT banding may reduce the incidence esophageal varices and/or bleeding from these varices even with close post-BRTO endoscopic follow-up.

research. Most studies display a gastric variceal rebleed rate of patients who had undergone a successful BRTO-procedure that ranges between zero and 20\% (all studies except one with a gastric rebleed rate under 10\%). ${ }^{3,11,12,25-32,35,41,87,88,90,92}$ However, when factoring in an intent-to-treat basis (including technical failures) for the results, the gastric variceal rebleed rate is zero to $31.6 \% .^{3,11,12,25-32,35,41,87,88,90,92}$ Many studies do not clearly state what, if any, is the global rebleed rate from: gastric, esophageal, duodenal varices as well as portal hypertensive gastropathy. ${ }^{3,11,12,26,28-32,35,41,87,88,92}$ In three clearly reported studies evaluating 141 patients who had undergone a BRTO procedure, the gastric variceal rebleed rate of successful BRTO procedures, the intent-to-treat 
gastric variceal rebleed rate, and the global (all types of varices) variceal rebleed rate was: $3.2-8.7 \%, 10-20 \%$, and $19-31 \%$, respectively. ${ }^{25,27,90}$ Compare these results with the latest four studies evaluating 87 patients who underwent a TIPS procedure with a 12-24 month global rebleed rate of $11-20 \%{ }^{26,79,82,83}$ Both sets of studies (BRTO vs TIPS) leave room for improvement. The BRTO studies probably can reduce rebleeding with aggressive endoscopic management and the TIPS studies probably can reduce rebleeding by utilizing stent grafts and a strict Doppler ultrasound follow-up.

Perhaps one of the greatest advantages of BRTO over TIPS is its preservation of hepatic function and its reduction in the risk of hepatic encephalopathy. In fact, one of the indications for BRTO is encephalopathy with the presence of a gastrorenal or gastrosplenorenal shunt. ${ }^{3,12,24,33-35,40,88}$ In five studies evaluating $35 \mathrm{pa}^{-}$ tients with encephalopathy, there was resolution or significant reduction in encephalopathy in all $(100 \%$ success) patients. ${ }^{3,12,33,35,88}$ The Kaplan-Meier survival rate after BRTO is impressive. The 1-, 2-, 3-, and 5-year survival rates range from $83-98 \%, 76-79 \%, 66-85 \%$, and 39-69\%, respectively. ${ }^{3,27,31,35,40-42,90}$ Obviously, the greatest determinate of survival is the patient's hepatic reserve (Child-Pugh score and/or MELD score). ${ }^{3,27,40,41}$ However, hepatocellular carcinoma (HCC) is also a significant determinate of survival ${ }^{35,40,41}$ to the extent that prior authors have considered an intrahepatic $\mathrm{HCC}$ of $>5 \mathrm{~cm}$ as a contraindication for BRTO. $^{35}$

\section{DISCUSSION}

Above is a brief literature-based synopsis of the history and clinical effectiveness of both the balloon-occluded transvenous obliteration (BRTO) and the transjugular intrahepatic portosystemic shunt (TIPS) procedures specific to managing gastric varices. The following discussion is based on our opinion with speculations and proposals on how gastric varices can possibly be managed by interventional radiologists (BRTO and/or TIPS) in the United States. The literature for both procedures leaves a lot to be desired and thus leaves a lot of room for speculation. For TIPS, an evaluation of the upper gastrointestinal rebleed rate for TIPS created by stent grafts is required. For BRTO, a completely disclosed upper gastrointestinal rebleed rate (not just from gastric varices) with adequate follow-up is required. In addition, the effectiveness of the BRTO procedure should be evaluated for patients with a poor hepatic reserve (MELD >17-19). To date, there is no evaluation of the effect of BRTO on the MELD score (there is for the Child-Pugh score) let alone stratification of hepatic function and survival based on the MELD score. Furthermore, we agree with Jalan and Hayes that a randomized controlled clinical trial comparing TIPS with BRTO is needed. ${ }^{93}$

We also agree with Jalan and Hayes and stress that the common reference to the 50\% rebleed rate following TIPS is incorrect, ${ }^{93}$ especially because it was based on bare-stent technology. This is the obliteration rate/lack of flow in the gastric varices $(N=6 / 12)$ following TIPS. ${ }^{85}$ Even when evaluating the gastric varix obliteration rate $(50 \%$ for TIPS and $75-100 \%$ for BRTO) is an uneven comparison. First, the two procedures are different. One procedure (BRTO) is a direct sclerotherapy of the varices and the other procedure (TIPS) is a shunt that competes with the blood flow within the varices. Second, and more importantly, rebleeding is what counts ultimately and is the true gauge of clinical success and not whether there is flow within the gastric varix. The latter is a technical/anatomical variable and not a clinical variable.

A not uncommon discussion in the BRTO literature is the ability of the BRTO procedure to divert portal blood flow toward the liver and thus improving, if not preserving, hepatic function. ${ }^{11,27,32,35,36,90}$ However, admittedly certain patients have preserved or improved hepatic function and others are unresponsive to the increased flow. ${ }^{32,90}$ Furthermore, the patients that do have an improved hepatic function return to baseline within 6 to 9 months (The improvement of hepatic function is transient). ${ }^{32,35,36}$ The theories to explain this include (1)gradual reduction in the hepatic vascular resistance, and/or (2)development of portosystemic shunts particularly the esophagoazygous route (esophageal varices) that auto-decompress the portal system. ${ }^{35}$ The hypothesis behind why certain patients do not respond to the portal flow diversion is that certain patients have irreversible hepatic disease/damage that does not respond to increased portal blood flow. ${ }^{32}$ However, all these studies neglect the fact that there is another major component of this blood flow diversion theory that has to be taken into account. This neglected factor is the potential variability in the amount of portal blood flow that is diverted. This is because not all gastrorenal or gastrosplenorenal have the same throughput that can potentially be diverted after a BRTO procedure. How can this throughput be gauged? (flow meters, Doppler ultrasound, perfusion MR). What is the portal pressure before and after the BRTO and can that be stratified to responders versus nonresponders? We find this to be a very interesting area of research that can be pivotal in the BRTO versus TIPS versus BRTO combined with TIPS debate. Do very large gastrorenal shunts with significant throughput, when blocked, divert large volumes of portal blood toward the liver causing a significant rise in the portal pressure particularly with severely diseased noncompliant livers? Do these high throughput gastrorenal shunts then require a TIPS to compensate for their sudden occlusion by a BRTO 
procedure in an attempt to prevent nongastric varices aggravation or ascites development? Can these TIPS be intentionally temporary just to last for 6 to 9 months (the period when the improved hepatic function returns to baseline). Is this the return of TIPS utilizing bare stents for this particular scenario? Conversely, when performing a TIPS and finding a portosystemic gradient of $12 \mathrm{~mm} \mathrm{Hg}$, does this signify a significant gastrorenal shunt that will not respond to TIPS shunting. Even more so, is this gastrorenal shunt so significant that it can act as a competing shunt with the TIPS and potentially lead to TIPS thrombosis with or without portal vein thrombosis? Remember, that $75 \%$ of unobliterated gastric varices had a pre-TIPS portosystemic gradient of less than $12 \mathrm{~mm} \mathrm{Hg} .{ }^{85}$ Anecdotally, a significant gastrorenal shunt is defined as a pre-TIPS portosystemic gradient of $<12 \mathrm{~mm} \mathrm{Hg}$ and/or hepatofugal (reversed) flow in the splenic vein with contrast escaping into the left renal vein despite a well placed and widely patent TIPS with a portosystemic gradient $<10-12 \mathrm{~mm} \mathrm{Hg}$.

In addition, to the throughput theory where some gastrorenal shunts have significant blood flow competing with the TIPS and decompressing the portal circulation rendering a pre-TIPS portosystemic gradient $<12 \mathrm{~mm}$ $\mathrm{Hg}$, there is another theory that may explain why TIPS may be less effective in decompressing gastric varices compared with esophageal varices. This theory is "the proximity theory" (our term). The portal feeder to the gastric varices is usually the posterior or short gastric vein(s), which are closer to the gastrorenal shunt (on the left side of the portal circulation) than they are to the TIPS (intrahepatic and in the right side of the portal circulation). Compare this with the usual portal feeder to the esophageal varices (coronary vein/left gastric vein). The left gastric vein originates in the right side of the portal circulation. In fact the distance between a TIPS and the portal origin of posterior gastric vein (and even more so, the short gastric vein) is approximately twice the distance compared with the distance between a TIPS and the portal origin of the left gastric vein. This brings the discussion to another anatomic variable that ideally should be taken into account. Gastroesophageal varices can be fed primarily from the coronary vein; and thus the gastric varix component may resolve with a TIPS. Possibly, a large number of the gastric varices that do respond to TIPS are actually complex gastroesophageal varices with dominant left gastric veins and poorly supplied by the more distant posterior and short gastric veins. Therefore, from a fluid mechanics standpoint, when hemodynamically discussing steal/sump/diversion flow phenomena it is not only the throughput between the competing shunts (in this case TIPS vs gastrorenal shunts), but their proximity to what they are "competing over" to decompress. The original surgical doctrine of surgically placed portosystemic shunts took note of this. $94-96$ If there was a "left-sided portal problem" (spleen of splenic vein), the best shunt would be a leftsided shunt (distal splenorenal shunt, for example) and if it was a right-sided or liver problem a central or rightsided shunt is more appropriate. ${ }^{94-96}$ The distal splenorenal shunt (in essence a surgically made left-side/ shunt somewhat equivalent to a spontaneous gastrorenal or gastrospleno renal shunt) is known to "compartmentalize" the portal circulation maintaining hepatopetal flow in the main portal vein due to a maintained high pressure along the mesoportal axis, while reducing the portal pressure for the left, posterior, and short gastric veins. ${ }^{94-96}$ The Warren-Salem distal splenorenal shunt was designed to maintain the hepatic function while decompressing the origins of gastroesophageal varices. ${ }^{94-96}$ It was proven to be ineffective for right-sided portal circulation (hepatic/sinusoidal) problems such as ascites. ${ }^{94-96}$ In the case of a TIPS it is the opposite. It is a right-sided shunt placed to decompress a left-sided portal circulation problem, i.e., gastric varices and their, not uncommonly associated, gastrorenal shunt.

Another common remark in the BRTO literature is that the BRTO procedure is "easier" than the TIPS procedure. ${ }^{93} \mathrm{We}$, who perform both these procedures routinely, disagree. They are largely non-comparable. They both are involved procedures requiring a different skill set and anatomic knowledge of different parts of the portal circulation; however, both can be quite challenging. We agree that BRTO is definitely less invasive.

When discussing the logistics and the utilization of hospital resources, each procedure's utilization of resources may differ from one institution to another and it is difficult to make a general remark about logistics. The first stage of the BRTO procedure usually does not require anesthesia and usually takes less than 1-2 hours in experienced hands. ${ }^{24-27,31-35}$ Subsequently, the balloon is retained for 6 to 24 hours (usually 6-12 hours) and the patient is to return for a fluoroscopic-guided balloon deflation and removal. ${ }^{24-42} \mathrm{On}$ the other hand, the TIPS procedure is a one-stage procedure and is also completed within 1-2 hours in experienced hands. However, some institutions do require the TIPS procedure to be done under general anesthesia. This raises the hospital resource requirements and increases the overall utilization of the angiography suite.

Until all of the above matters are clarified, one can only speculate as to what is the better option in particular patients with portal hypertensive complications. TIPS is probably the better option for a patient with gastric varices and refractory ascites because it manages portal hypertension globally. BRTO can definitely be resorted to when patients are not TIPS candidates. This population is not necessarily small. This includes patients who are encephalopathic at baseline, have a poor hepatic reserve (MELD >17-19), have had a failed TIPS 
procedure, have active gastric variceal bleeding with intractable coagulopathy, have had prior lobar chemoembolizations or patients with HCC who can be candidates for subsequent chemoembolization. The latter should be evaluated as a global hepatobiliary and oncology consult: BRTO with subsequent chemoembolization versus TIPS and other alternative systemic treatments or percutaneous tumor ablation. Anecdotally, a patient with HCC that is not a chemoembolization candidate (bilirubin $>3.0 \mathrm{mg} /$ deciliter) is usually not going to be a TIPS candidate to begin with (more likely has a high MELD score). A patient with hepatic encephalopathy, ascites, and gastric varices probably has a poor hepatic reserve and a poor outcome regardless of what would be done, if anything at all.

\section{CONCLUSION}

Both the TIPS and the BRTO procedures are valuable procedures in the armamentarium of interventional radiologists. They require a different skill-set and they have proven to be effective in managing gastric varices. A lot of research work is required to determine the effectiveness of one procedure over the other especially in particular clinical scenarios. Ideally, in the future, patients with gastric varices would be stratified according to(1)the severity of portal hypertension and the size of the gastrorenal shunt throughput in unison, (2)other complications of liver cirrhosis and/or portal hypertension (HCC, ascites, encephalopathy), and (3)hepatic reserve to undergo the ideal procedure (BRTO vs TIPS vs both). Until this research is performed adequately, the ideal BRTO versus TIPS candidate is speculative. Having said that, BRTO most likely is a procedure that preserves the hepatic function; thus, patients with poor hepatic reserve along with other non-TIPS candidates should be triaged to undergo a BRTO procedure if it is feasible (if a gastro-renal shunt exists).

\section{REFERENCES}

1. Ryan BM, Stockbrugger RW, Ryan JM. A pathophysiologic, gastroenterologic, and radiologic approach to the management of gastric varices. Gastroenterology 2004;126(4): $1175-1189$

2. Sarin SK, Lahoti D, Saxena SP, Murthy NS, Makwana UK. Prevalence, classification and natural history of gastric varices: a long-term follow-up study in 568 portal hypertension patients. Hepatology 1992;16(6):1343-1349

3. Fukuda T, Hirota S, Sugimura K. Long-term results of balloon-occluded retrograde transvenous obliteration for the treatment of gastric varices and hepatic encephalopathy. J Vasc Interv Radiol 2001;12(3):327-336

4. Trudeau W, Prindiville T. Endoscopic injection sclerosis in bleeding gastric varices. Gastrointest Endosc 1986;32(4): 264-268
5. Korula J, Chin K, Ko Y, Yamada S. Demonstration of two distinct subsets of gastric varices. Observations during a seven-year study of endoscopic sclerotherapy. Dig Dis Sci 1991;36(3):303-309

6. Oho K, Iwao T, Sumino M, Toyonaga A, Tanikawa K. Ethanolamine oleate versus butyl cyanoacrylate for bleeding gastric varices: a nonrandomized study. Endoscopy 1995; 27(5):349-354

7. Kim HG, Han KH, Lee CY, et al. Outcome of endoscopic injection therapy of histoacryl in bleeding gastric varices. Gastroenterology 1998;114:A1273

8. Rockey DC. Management of gastric varices. Gastroenterology 2001;120(7):1875-1876; discussion 1876-1877

9. Kiyosue H, Mori H, Matsumoto S, Yamada Y, Hori Y, Okino Y. Transcatheter obliteration of gastric varices. Part 1. Anatomic classification. Radiographics 2003;23(4): 911-920

10. Kiyosue H, Mori H, Matsumoto S, Yamada Y, Hori Y, Okino Y. Transcatheter obliteration of gastric varices: Part 2. Strategy and techniques based on hemodynamic features. Radiographics 2003;23(4):921-937; discussion 937

11. Ibukuro K, Sugihara T, Tanaka R, et al. Balloon-occluded retrograde transvenous obliteration (BRTO) for a direct shunt between the inferior mesenteric vein and the inferior vena cava in a patient with hepatic encephalopathy. J Vasc Interv Radiol 2007;18(1 Pt 1):121-125

12. Ninoi T, Nishida N, Kaminou T, et al. Balloon-occluded retrograde transvenous obliteration of gastric varices with gastrorenal shunt: long-term follow-up in 78 patients. AJR Am J Roentgenol 2005;184(4):1340-1346

13. Hirota S, Matsumoto S, Tomita M, Sako M, Kono M. Retrograde transvenous obliteration of gastric varices. Radiology 1999;211(2):349-356

14. Kanagawa H, Mima S, Kouyama H, Gotoh K, Uchida T, Okuda K. Treatment of gastric fundal varices by balloonoccluded retrograde transvenous obliteration. J Gastroenterol Hepatol 1996;11(1):51-58

15. Hayashi S, Saeki S, Hosoi H, et al. A clinical and portal hemodynamic analysis for obliteration of gastric-renal shunt communicated with gastric fundic varices. Nippon Shokakibyo Gakkai Zasshi 1998;95(7):755-763

16. Tanihata $\mathrm{H}$, Minamiguchi $\mathrm{H}$, Sato $\mathrm{M}$, et al. Changes in portal systemic pressure gradient after balloon-occluded retrograde transvenous obliteration of gastric varices and aggravation of esophageal varices. Cardiovasc Intervent Radiol 2009;32(6):1209-1216

17. Choi YS, Lee JH, Sinn DH, et al. Effect of balloon-occluded retrograde transvenous obliteration on the natural history of coexisting esophageal varices. J Clin Gastroenterol 2008; 42(9):974-979

18. Nakamura S, Torii N, Yatsuji S, et al. Long-term follow up of esophageal varices after balloon-occluded retrograde transvenous obliteration for gastric varices. Hepatol Res 2008;38(4):340-347

19. Funaro AH, Ring EJ, Freiman DB, Oleaga JA, Gordon RL. Transhepatic obliteration of esophageal varices using the stainless steel coil. AJR Am J Roentgenol 1979;133(6): 1123-1125

20. Scott J, Dick R, Long RG, Sherlock S. Percutaneous transhepatic obliteration of gastro-oesophageal varices. Lancet 1976;2(7976):53-55

21. Lunderquist A, Simert G, Tylén U, Vang J. Follow-up of patients with portal hypertension and esophageal varices 
treated with percutaneous obliteration of gastric coronary vein. Radiology 1977;122(1):59-63

22. Lunderquist A, Vang J. Transhepatic catheterization and obliteration of the coronary vein in patients with portal hypertension and esophageal varices. N Engl J Med 1974;291(13):646-649

23. Lunderquist A, Vang J. Sclerosing injection of esophageal varices through transhepatic selective catheterization of the gastric coronary vein. A preliminary report. Acta Radiol Diagn (Stockh) 1974;15(5):546-550

24. Olson E, Yune HY, Klatte EC. Transrenal-vein reflux ethanol sclerosis of gastroesophageal varices. AJR Am J Roentgenol 1984;143(3):627-628

25. Yamagami T, Kato T, Hirota T, Yoshimatsu R, Matsumoto $\mathrm{T}$, Nishimura T. Infusion of $50 \%$ glucose solution before injection of ethanolamine oleate during balloon-occluded retrograde transvenous obliteration. Australas Radiol 2007; 51(4):334-338

26. Kitamoto M, Imamura M, Kamada K, et al. Balloonoccluded retrograde transvenous obliteration of gastric fundal varices with hemorrhage. AJR Am J Roentgenol 2002; 178(5):1167-1174

27. Ninoi $T$, Nakamura $K$, Kaminou $T$, et al. TIPS versus transcatheter sclerotherapy for gastric varices. AJR Am J Roentgenol 2004;183(2):369-376

28. Akahoshi T, Hashizume M, Tomikawa M, et al. Long-term results of balloon-occluded retrograde transvenous obliteration for gastric variceal bleeding and risky gastric varices: a 10-year experience. J Gastroenterol Hepatol 2008;23(11): 1702-1709

29. Sonomura T, Sato M, Kishi K, et al. Balloon-occluded retrograde transvenous obliteration for gastric varices: a feasibility study. Cardiovasc Intervent Radiol 1998;21(1): $27-30$

30. Kiyosue H, Matsumoto S, Onishi R, et al. Balloon-occluded retrograde transvenous obliteration (B-RTO) for gastric varices: therapeutic results and problems. Nippon Igaku Hoshasen Gakkai Zasshi 1999;59(1):12-19

31. Koito K, Namieno T, Nagakawa T, Morita K. Balloonoccluded retrograde transvenous obliteration for gastric varices with gastrorenal or gastrocaval collaterals. AJR Am J Roentgenol 1996;167(5):1317-1320

32. Chikamori F, Kuniyoshi N, Kawashima T, Takase Y. Gastric varices with gastrorenal shunt: combined therapy using transjugular retrograde obliteration and partial splenic embolization. AJR Am J Roentgenol 2008;191(2):555-559

33. Kumamoto $M$, Toyonaga $A$, Inoue $H$, et al. Long-term results of balloon-occluded retrograde transvenous obliteration for gastric fundal varices: hepatic deterioration links to portosystemic shunt syndrome. J Gastroenterol Hepatol 2010;25(6):1129-1135

34. Chikamori F, Kuniyoshi N, Shibuya S, Takase Y. Transjugular retrograde obliteration for chronic portosystemic encephalopathy. Abdom Imaging 2000;25(6):567-571

35. Chikamori F, Kuniyoshi N, Shibuya S, Takase Y. Combination treatment of transjugular retrograde obliteration and endoscopic embolization for portosystemic encephalopathy with esophageal varices. Hepatogastroenterology 2004; 51(59):1379-1381

36. Chikamori F, Kuniyoshi N, Shibuya S, Takase Y. Eight years of experience with transjugular retrograde obliteration for gastric varices with gastrorenal shunts. Surgery 2001;129(4): 414-420
37. Miyamoto Y, Oho K, Kumamoto M, Toyonaga A, Sata M. Balloon-occluded retrograde transvenous obliteration improves liver function in patients with cirrhosis and portal hypertension. J Gastroenterol Hepatol 2003;18(8): 934-942

38. Sugimori K, Morimoto M, Shirato K, et al. Retrograde transvenous obliteration of gastric varices associated with large collateral veins or a large gastrorenal shunt. J Vasc Interv Radiol 2005;16(1):113-118

39. Fukuda T, Hirota S, Matsumoto S, et al. Application of balloon-occluded retrograde transvenous obliteration to gastric varices complicating refractory ascites. Cardiovasc Intervent Radiol 2004;27(1):64-67

40. Shimoda R, Horiuchi K, Hagiwara S, et al. Short-term complications of retrograde transvenous obliteration of gastric varices in patients with portal hypertension: effects of obliteration of major portosystemic shunts. Abdom Imaging 2005;30(3):306-313

41. Takuma Y, Nouso K, Makino Y, Saito S, Shiratori Y. Prophylactic balloon-occluded retrograde transvenous obliteration for gastric varices in compensated cirrhosis. Clin Gastroenterol Hepatol 2005;3(12):1245-1252

42. Arai H, Abe T, Takagi H, Mori M. Efficacy of balloonoccluded retrograde transvenous obliteration, percutaneous transhepatic obliteration and combined techniques for the management of gastric fundal varices. World J Gastroenterol 2006;12(24):3866-3873

43. Arai H, Abe T, Shimoda R, Takagi H, Yamada T, Mori M. Emergency balloon-occluded retrograde transvenous obliteration for gastric varices. J Gastroenterol 2005;40(10): 964-971

44. Burgener FA, Gutierrez OH. Nonsurgical production of intrahepatic portosystemic venous shunts in portal hypertension with the double lumen balloon catheter (author's transl). Rofo 1979;130(6):686-688

45. Burgener FA, Gutierrez OH. Production of an intrahepatic portocaval fistula in the dog with liver cirrhosis and portal hypertension. Rofo 1984;141(3):327-332

46. Burgener FA, Gutierrez OH. Production of nonsurgical intrahepatic portacaval shunt in portal hypertension using a Gruntzig balloon dilatation catheter. J Radiol 1985;66(1): 7-11

47. Burgener FA, Gutierrez OH. Experimental intrahepatic portocaval shunts created in portal hypertension by balloon angioplasty catheters. Invest Radiol 1988;23(1):24-29

48. Rösch J, Uchida BT, Putnam JS, Buschman RW, Law RD, Hershey AL. Experimental intrahepatic portacaval anastomosis: use of expandable Gianturco stents. Radiology 1987; 162(2):481-485

49. LaBerge JM, Ring EJ, Gordon RL. Percutaneous intrahepatic portosystemic shunt created via a femoral vein approach. Radiology 1991;181(3):679-681

50. Ring EJ, Lake JR, Roberts JP, et al. Using transjugular intrahepatic portosystemic shunts to control variceal bleeding before liver transplantation. Ann Intern Med 1992;116(4): 304-309

51. LaBerge JM, Ring EJ, Lake JR, et al. Transjugular intrahepatic portosystemic shunts: preliminary results in 25 patients. J Vasc Surg 1992;16(2):258-267

52. Sahagun G, Benner KG, Saxon R, et al. Outcome of 100 patients after transjugular intrahepatic portosystemic shunt for variceal hemorrhage. Am J Gastroenterol 1997;92(9): 1444-1452 
53. LaBerge JM, Ring EJ, Gordon RL, et al. Creation of transjugular intrahepatic portosystemic shunts with the wallstent endoprosthesis: results in 100 patients. Radiology 1993;187(2):413-420

54. Echenagusia AJ, Camúñez F, Simó G, et al. Variceal hemorrhage: efficacy of transjugular intrahepatic portosystemic shunts created with Strecker stents. Radiology 1994; 192(1):235-240

55. Sterling KM, Darcy MD. Stenosis of transjugular intrahepatic portosystemic shunts: presentation and management. AJR Am J Roentgenol 1997;168(1):239-244

56. Saxon RS, Ross PL, Mendel-Hartvig J, et al. Transjugular intrahepatic portosystemic shunt patency and the importance of stenosis location in the development of recurrent symptoms. Radiology 1998;207(3):683-693

57. Haskal ZJ, Pentecost MJ, Soulen MC, Shlansky-Goldberg $\mathrm{RD}$, Baum RA, Cope C. Transjugular intrahepatic portosystemic shunt stenosis and revision: early and midterm results. AJR Am J Roentgenol 1994;163(2):439-444

58. Nazarian GK, Bjarnason H, Dietz CA Jr, et al. Refractory ascites: midterm results of treatment with a transjugular intrahepatic portosystemic shunt. Radiology 1997;205(1): 173-180

59. Saxon RR, Timmermans HA, Uchida BT, et al. Stent-grafts for revision of TIPS stenoses and occlusions: a clinical pilot study. J Vasc Interv Radiol 1997;8(4):539-548

60. Haskal ZJ, Davis A, McAllister A, Furth EE. PTFEencapsulated endovascular stent-graft for transjugular intrahepatic portosystemic shunts: experimental evaluation. Radiology 1997;205(3):682-688

61. Haskal ZJ. Improved patency of transjugular intrahepatic portosystemic shunts in humans: creation and revision with PTFE stent-grafts. Radiology 1999;213(3):759-766

62. Otal P, Smayra T, Bureau C, et al. Preliminary results of a new expanded-polytetrafluoroethylene-covered stent-graft for transjugular intrahepatic portosystemic shunt procedures. AJR Am J Roentgenol 2002;178(1):141-147

63. Cejna M, Peck-Radosavljevic M, Thurnher SA, Hittmair K, Schoder M, Lammer J. Creation of transjugular intrahepatic portosystemic shunts with stent-grafts: initial experiences with a polytetrafluoroethylene-covered nitinol endoprosthesis. Radiology 2001;221(2):437-446

64. Cejna M, Peck-Radosavljevic M, Thurnher S, et al. ePTFEcovered stent-grafts for revision of obstructed transjugular intrahepatic portosystemic shunt. Cardiovasc Intervent Radiol 2002;25(5):365-372

65. Angermayr B, Cejna M, Koenig F, et al; Vienna TIPS Study Group. Survival in patients undergoing transjugular intrahepatic portosystemic shunt: ePTFE-covered stentgrafts versus bare stents. Hepatology 2003;38(4):1043-1050

66. Bureau C, Garcia-Pagan JC, Otal P, et al. Improved clinical outcome using polytetrafluoroethylene-coated stents for TIPS: results of a randomized study. Gastroenterology 2004;126(2):469-475

67. Angeloni S, Merli M, Salvatori FM, et al. Polytetrafluoroethylene-covered stent grafts for TIPS procedure: 1-year patency and clinical results. Am J Gastroenterol 2004;99(2): 280-285

68. Rossi P, Salvatori FM, Fanelli F, et al. Polytetrafluoroethylene-covered nitinol stent-graft for transjugular intrahepatic portosystemic shunt creation: 3-year experience. Radiology 2004;231(3):820-830
69. Charon J-PM, Alaeddin FH, Pimpalwar SA, et al. Results of a retrospective multicenter trial of the Viatorr expanded polytetrafluoroethylene-covered stent-graft for transjugular intrahepatic portosystemic shunt creation. J Vasc Interv Radiol 2004;15(11):1219-1230

70. Hausegger KA, Karnel F, Georgieva B, et al. Transjugular intrahepatic portosystemic shunt creation with the Viatorr expanded polytetrafluoroethylene-covered stent-graft. J Vasc Interv Radiol 2004;15(3):239-248

71. Vignali C, Bargellini I, Grosso M, et al. TIPS with expanded polytetrafluoroethylene-covered stent: results of an Italian multicenter study. AJR Am J Roentgenol 2005;185(2): 472-480

72. Rössle M, Siegerstetter V, Euringer W, et al. The use of a polytetrafluoroethylene-covered stent graft for transjugular intrahepatic portosystemic shunt (TIPS): Long-term followup of 100 patients. Acta Radiol 2006;47(7):660-666

73. Tripathi D, Ferguson J, Barkell H, et al. Improved clinical outcome with transjugular intrahepatic portosystemic stentshunt utilizing polytetrafluoroethylene-covered stents. Eur J Gastroenterol Hepatol 2006;18(3):225-232

74. Bureau C, Pagan JC, Layrargues GP, et al. Patency of stents covered with polytetrafluoroethylene in patients treated by transjugular intrahepatic portosystemic shunts: long-term results of a randomized multicentre study. Liver Int 2007; 27(6):742-747

75. Saxon RR. A new era for transjugular intrahepatic portosystemic shunts? J Vasc Interv Radiol 2004;15(3):217-219

76. Ferral H, Gamboa P, Postoak DW, et al. Survival after elective transjugular intrahepatic portosystemic shunt creation: prediction with model for end-stage liver disease score. Radiology 2004;231(1):231-236

77. Ferral H, Patel NH. Selection criteria for patients undergoing transjugular intrahepatic portosystemic shunt procedures: current status. J Vasc Interv Radiol 2005;16(4): 449-455

78. Montgomery A, Ferral H, Vasan R, Postoak DW. MELD score as a predictor of early death in patients undergoing elective transjugular intrahepatic portosystemic shunt (TIPS) procedures. Cardiovasc Intervent Radiol 2005;28(3):307-312

79. Anderson CL, Saad WE, Kalagher SD, et al. Effect of transjugular intrahepatic portosystemic shunt placement on renal function: a 7-year, single-center experience. J Vasc Interv Radiol 2010;21(9):1370-1376

80. Choi YH, Yoon CJ, Park JH, Chung JW, Kwon JW, Choi GM. Balloon-occluded retrograde transvenous obliteration for gastric variceal bleeding: its feasibility compared with transjugular intrahepatic portosystemic shunt. Korean J Radiol 2003;4(2):109-116

81. Chau TN, Patch D, Chan YW, Nagral A, Dick R, Burroughs AK. "Salvage" transjugular intrahepatic portosystemic shunts: gastric fundal compared with esophageal variceal bleeding. Gastroenterology 1998;114(5):981-987

82. Barange $\mathrm{K}$, Péron J-M, Imani $\mathrm{K}$, et al. Transjugular intrahepatic portosystemic shunt in the treatment of refractory bleeding from ruptured gastric varices. Hepatology 1999;30(5):1139-1143

83. Lo G-H, Liang $\mathrm{H}-\mathrm{L}$, Chen $\mathrm{W}-\mathrm{C}$, et al. A prospective, randomized controlled trial of transjugular intrahepatic portosystemic shunt versus cyanoacrylate injection in the prevention of gastric variceal rebleeding. Endoscopy 2007; 39(8):679-685 
84. Rees CJ, Nylander DL, Thompson NP, Rose JD, Record $\mathrm{CO}$, Hudson M. Do gastric and oesophageal varices bleed at different portal pressures and is TIPS an effective treatment? Liver 2000;20(3):253-256

85. Saad WEA, Darwish WM, Davies MG, Waldman DL. Stent-grafts for transjugular intrahepatic portosystemic shunt creation: specialized TIPS stent-graft versus generic stentgraft/bare stent combination. J Vasc Interv Radiol 2010; 21(10):1512-1520

86. Sanyal AJ, Freedman AM, Luketic VA, et al. The natural history of portal hypertension after transjugular intrahepatic portosystemic shunts. Gastroenterology 1997;112(3):889898

87. Chao Y, Lin HC, Lee FY, et al. Hepatic hemodynamic features in patients with esophageal or gastric varices. J Hepatol 1993;19(1):85-89

88. Hong $\mathrm{CH}$, Kim HJ, Park JH, et al. Treatment of patients with gastric variceal hemorrhage: endoscopic N-butyl-2cyanoacrylate injection versus balloon-occluded retrograde transvenous obliteration. J Gastroenterol Hepatol 2009; 24(3):372-378

89. Park KS, Kim YH, Choi JS, et al. Therapeutic efficacy of balloon-occluded retrograde transvenous obliteration in patients with gastric variceal bleeding. Korean J Gastroenterol 2006;47(5):370-378

90. Kim ES, Park SY, Kwon KT, et al. The clinical usefulness of balloon occluded retrograde transvenous obliteration in gastric variceal bleeding. Taehan Kan Hakhoe Chi 2003; 9(4):315-323

91. Cho SK, Shin SW, Lee IH, et al. Balloon-occluded retrograde transvenous obliteration of gastric varices: outcomes and complications in 49 patients. AJR Am J Roentgenol 2007;189(6):W365-72

92. Park SJ, Chung JW, Kim H-C, Jae HJ, Park JH. The prevalence, risk factors, and clinical outcome of balloon rupture in balloon-occluded retrograde transvenous obliteration of gastric varices. J Vasc Interv Radiol 2010;21(4): 503-507

93. Matsumoto A, Hamamoto N, Nomura T, et al. Balloonoccluded retrograde transvenous obliteration of high risk gastric fundal varices. Am J Gastroenterol 1999;94(3): 643-649

94. Jalan R, Hayes PC. Reply to: Management of gastric fundal varices associated with a gastrorenal shunt, written by Matsumoto A, Matsumoto H, Hamamoto N, Kayazawa M. Gut 2001;48:440-441

95. Knechtle SJ, Rikkers LF. The place of portosystemic shunting. In: Blumgart LH, Belghiti J, DeMatteo RP, et al, eds. Surgery of the Liver, Biliary Tract, and Pancreas. 4th ed. Vol. 2. Philadelphia, PA: Saunders Elsevier; 2007

96. Henderson JM. Distal splenorenal shunt. In: Blumgart LH, Belghiti J, DeMatteo RP, et al, eds. Surgery of the Liver, Biliary Tract, and Pancreas. 4th ed. Vol. 2. Philadelphia, PA: Saunders Elsevier; 2007 